\title{
Clinical Aspects of Screening Test Tools for Central Neuropathic Pain in Patients with Thalamic Stroke
}

\author{
In Hee Lee, PT, $\mathrm{PhD}^{1)}$, Yoon Nyun Kim, MD, $\mathrm{PhD}^{2)}$, Chang Sik Son, $\mathrm{PhD}^{3)}$, \\ Yong Hyun Kwon, PT, $\mathrm{PhD}^{4)}$, Min Soo Kim, $\mathrm{PhD}^{5)}$, SuK TaE SEO, PhD ${ }^{5)}$ \\ 1) Department of Physical Medicine and Rehabilitation, Dongsan Hospital, Keimyung University \\ 2) Department of Internal Medicine, School of Medicine, Keimyung University \\ ${ }^{3)}$ Department of Medical Informatics, School of Medicine, Keimyung University \\ 4) Department of Physical Therapy, Yeungnam College of Science \& Technology \\ ${ }^{5)}$ Biomedical Information Technology Center, Keimyung University: \\ 1000 Sindang-dong Dalseo-Gu, Daegu,704-701, Korea.E-mail : kenneth78@kmu.ac.kr
}

\begin{abstract}
Purpose] The purpose of this study was to investigate clinical aspects of screen test tools for central neuropathic pain in thalamic stroke patients. [Subjects and Methods] Seven thalamic stroke patients were recruited as subjects. To classify the subjects into central neuropathic pain and non-neuropathic pain groups, the Leeds assessment of neuropathic symptoms and signs (LANSS) was used. Four patients were classified as having central neuropathic pain. To evaluate the central neuropathic pain, the quantitative somatosensory test, the median nerve somatosensory evoked potentials (SEPs), magnetic resonance imaging (MRI), and functional MRI (fMRI) were performed on average 31.5 months after stroke. [Results] The quantitative somatosensory test did not show a correlation between the central neuropathic pain group and the non-neuropathic pain group. The SEPs on the affected side showed a response in one of the patients without central neuropathic pain, and responses on the unaffected side were normal for all of the patients. MRI-based thalamic localization data indicate that this method is limited in its ability to distinguish the central neuropathic pain in thalamic stroke patients. Results of fMRI show that the secondary somatosensory areas of the central neuropathic pain group were more activated than those of nonneuropathic pain group. [Conclusion] Based on the results, we verified that functional MRI is useful for evaluating the central neuropathic pain in thalamic stroke patients.

Key words: Functional Magnetic Resonance Imaging, Neuropathic Pain, Stroke
\end{abstract}

(This article was submitted Apr. 1, 2011, and was accepted May. 10, 2011)

\section{INTRODUCTION}

Central post-stroke pain is characterized by sensory abnormalities that are evoked by non painful stimuli such as heat, cold or tactile stimuli ${ }^{1,2)}$. In Bowsher's report (1993), $2 \%$ of 400 stroke patients showed neuropathic pain, and $25 \%$ of 400 stroke patients had some somatosensory deficits $^{3)}$.

Thalamic lesions can lead not only to motor deficit but also to somatosensory deficiency and central neuropathic pain. Prognosis after thalamic lesion is generally regarded as being rather good compared with lesions of the cerebral cortex or other subcortical structures. However, this prognosis is generally the result of the low incidence of mortality and good recovery from motor deficit. Thalamic pain syndrome due to pathologic changes of the lateral thalamus is characterized by slight hemiplegia, disturbance of superficial and deep sensibility, hemiataxia, hemistereoagnosia, and intolerable pain ${ }^{4}$.

In previous studies of quantitative somatic sensory tests for patients with central post-stroke pain, thermal and pain sensations, which are transferred through the spinothalamic tract and/or thalamocortical structures, were decreased ${ }^{5,6)}$.
There are several reports about central post-stroke pain with thalamus lesion ${ }^{7,8)}$. However, there have been investigations of alterations to the cerebral cortex with thalamic stroke, which have been based on medical imaging such as functional magnetic resonance imaging (fMRI). Thus, the purpose of this study was to distinguish between central neuropathic pain and non-central neuropathic pain based on the Leeds assessment of neuropathic symptoms and signs (LANSS) pain scale and to observe the variation in cortical representation according to central neuropathic pain based on fMRI. The LANSS pain scale is a very useful screening tool for evaluating whether or not the nerves that are carrying pain signals are working normally or not. The LANSS pain scale is based on analysis of sensory description and bedside examination of sensory dysfunction and it is able to correctly identify $82 \%, 85 \%$ sensitivity and $80 \%$ specificity ${ }^{9}$.

The purpose of this study was to evaluate the usefulness of methods for screening central neuropathic pain in thalamus stroke patients. To evaluate them, LANSS was used to assess seven subjects with thalamic stroke, and they were classified into a central neuropathic pain group and a non-central neuropathic pain group based on the score of 
LANSS. Moreover, to investigate the differences between the groups, the results of the quantitative somatic sensory test, somatosensory evoked potentials (SEPs), magnetic resonance imaging (MRI), and fMRI were compared.

\section{SUBJECTS AND METHODS}

Seven stroke patients (three males and four females) with thalamic lesions were recruited for the study. All of the patients were enrolled in this study after providing their informed consent in accordance with the ethical standards of the Declaration of Helsinki. General information and lesion information of the subjects are summarized in Table 1. The subjects had no neurologic or psychiatric history before stroke according to their medical records and brain MRI. All of the enrolled subjects provided their written, informed consent prior to this experiment.

To evaluate quantitative somatic sensory, various tests, such as vibration, temperature sensory, point discrimination test, pin prick test, etc., were performed on the thumbs of the affected side, and the test results are summarized in Table 2.

SEPs were evoked by electrical stimulation $\left(\right.$ Synergy $^{\circledR}$, Medelec) of the median nerve in both wrists at a temperature of $22^{\circ} \mathrm{C} \sim 24^{\circ} \mathrm{C}$. Three micrographic contraction of the thenar muscles (test conditions: 3 time/sec, $300 \mathrm{~Hz}$, and $0.1 \mathrm{msec}$ duration) were performed. SEPs were recorded from scalp electrodes positioned over C3'/C4', referenced to $\mathrm{FZ}$, in accordance with the international 10 to 20 system (frequency range $3 \mathrm{~Hz} \sim 3 \mathrm{KHz}$, recording velocity $5 \mathrm{msec} /$ division, recording sensitivity $5 \mu \mathrm{V} /$ division). In this study, the presence of normal N20 and delayed latency was regarded as positive, and the absence of N20 was regarded as negative.

To evaluate the damaged region of the stroke patients, fMRI was performed. A blocked trial design, repeating tasks in time, was used to stimulate the median nerve for brain activation. The electrical stimulation of the median nerve was performed by Walking Man II (Cybermedic ${ }^{\circledR}$ ) with surface electrode wrists (frequency $30 \mathrm{~Hz}$, duration
Table 1. General characteristics of participants

\begin{tabular}{lc}
\hline Indexes & Values \\
\hline Age (Year) & $63.8 \pm 28.5$ (mean \pm standard deviation) \\
Gender (Male/Female) & $3 / 4$ \\
Infarction/Hemorrhage & $4 / 3$ \\
Affected Side (Right/Left) & $3 / 4$ \\
Mean Duration (Months) & $31.5 \pm 28.5$ (mean \pm standard deviation)
\end{tabular}

$200 \mu \mathrm{s})$. The intensity was below the contraction level of the thenar muscle. Three rest periods was alternated with three stimulation periods. Each period had a duration of the 20 seconds. Stimulation images were acquired during 2 minutes and they were compared with the rest period images.

The functional and anatomical imaging was performed on a 3.0 Tesla clinical MRI scanner (Signa VH/1, GE Medical Systems, USA) with surface electrodes. T2weighted gradient echo planar images (TR/TE 1900/40 msec, acquisition matrix $64 \times 64$, field of view (FOV) 240 $\mathrm{mm}$, number of excitation (NEX) 1, Slice thickness $5 \mathrm{~mm}$ ) were obtained for anatomical brain images. Blood oxygenation level dependent (BOLD) function images were also acquired (TR/TE 4500/104 msec, acquisition matrix $256 \times 256$, FOV $240 \mathrm{~mm}$, NEX 1 slice thickness $5 \mathrm{~mm}$ ).

The fMRI images were realigned using SPM-99 software (Welcome Department of Cognitive Neurology, London, UK) running under the MATLAB environment (The Mathworks, Inc., Natick, Ma, USA) after head motion correction, and functional images and anatomical images were co-registered. The brain activation difference between the rest and stimulation periods were tested by one-way ANOVA and voxels were considered significant at a threshold of $p<0.001$. Statistical analysis was conducted using SPSS 17.0, and $\mathrm{p}<0.05$ was adopted as the criterion for statistical significance.

Table 2. The response results of quantitative somatic sensory tests

\begin{tabular}{|c|c|c|c|c|}
\hline Indexes & Method & Responses & Neuropathic Pain & Non-neuropathic Pain \\
\hline \multirow{2}{*}{ Vibration } & \multirow{2}{*}{ Tuning fork } & No response & 1 & 1 \\
\hline & & Response & 3 & 2 \\
\hline \multirow{2}{*}{ Thermal warm } & \multirow{2}{*}{ Test tube $\left(40{ }^{\circ} \mathrm{C}\right)$} & No response & 1 & 3 \\
\hline & & Response & 3 & 0 \\
\hline \multirow{2}{*}{ Thermal cold } & \multirow{2}{*}{ Test tube $\left(20^{\circ} \mathrm{C}\right)$} & No response & 2 & 1 \\
\hline & & Response & 2 & 2 \\
\hline \multirow{2}{*}{ Stereognosis } & \multirow{2}{*}{ Clip on thumb and index } & No response & 2 & 3 \\
\hline & & Response & 2 & 0 \\
\hline \multirow{2}{*}{ Dermographics } & \multirow{2}{*}{ Figures on thumb } & No response & 2 & 2 \\
\hline & & Response & 2 & 1 \\
\hline \multirow{2}{*}{ Position } & \multirow{2}{*}{ Joint position of thumb } & No response & 1 & 3 \\
\hline & & Response & 3 & 0 \\
\hline \multirow{2}{*}{ Mechanical stimulation } & \multirow{2}{*}{ Von Frey filament } & No response & 0 & 2 \\
\hline & & Response & 4 & 1 \\
\hline 2 point Discrimination $(\mathrm{mm})$ & 2 point discriminator & & $366.2 \pm 435.9$ & $319.2 \pm 435.7$ \\
\hline Pin-prick (g) & perceived weight from $0.2 \mathrm{~g}$ to $5 \mathrm{~g}$ & & $1.44 \pm 1.16$ & $3.33 \pm 1.53$ \\
\hline
\end{tabular}


Table 3. The response results of somatosensory evoked potentials

\begin{tabular}{lcccc}
\hline \multirow{2}{*}{ SEPs } & \multicolumn{2}{c}{ Neuropathic pain group } & \multicolumn{2}{c}{ Non-neuropathic pain group } \\
\cline { 2 - 5 } & $\begin{array}{c}\text { Affected side } \\
\text { stimulation }\end{array}$ & $\begin{array}{c}\text { Unaffected side } \\
\text { Stimulation }\end{array}$ & $\begin{array}{c}\text { Affected side } \\
\text { stimulation }\end{array}$ & $\begin{array}{c}\text { Unaffected side } \\
\text { stimulation }\end{array}$ \\
\hline Response & 4 & 4 & 1 & 3 \\
No response & 4 & & 2 & \\
\hline
\end{tabular}

Table 4. Activation pattern of cortical areas during median nerve electrical stimulation

\begin{tabular}{|c|c|c|c|c|}
\hline \multirow[b]{2}{*}{ Activation of Cortical Area } & \multicolumn{2}{|c|}{ Neuropathic Pain } & \multicolumn{2}{|c|}{ Non-neuropathic Pain } \\
\hline & $\begin{array}{c}\text { Affected side } \\
\text { stimulation }\end{array}$ & $\begin{array}{l}\text { Unaffected } \\
\text { stimulation }\end{array}$ & $\begin{array}{c}\text { Affected } \\
\text { stimulation }\end{array}$ & $\begin{array}{l}\text { Unaffected } \\
\text { stimulation }\end{array}$ \\
\hline Ipsilateral SI & & & 2 & \\
\hline Ipsilateral SII & 2 & 1 & & \\
\hline Contralateral SI & & 1 & & 1 \\
\hline Contralateral SII & 1 & & 1 & \\
\hline Bilateral SI & & 1 & & \\
\hline Bilateral SII & 2 & 1 & 1 & 3 \\
\hline
\end{tabular}

The values are activation numbers, and SI and SII represent primary somatosensory cortex and secondary somatosensory cortex, respectively.

\section{RESULTS}

The results of the quantitative somatic sensory tests did not show any significant differences.

The results of SEPs are summarized in Table 3. All of the patients showed response on the unaffected side, and one patient from the non-central neuropathic pain group showed response on the affected side.

The results of fMRI analyzing the activation patterns are summarized in Table 4 and Figure 1.

In the affected side median nerve stimulation test, two patients showed activation of the ipisilateral secondary somatosensory cortex SII, one patient of the contralateral SII, and two patients of the bilateral SII in the central neuropathic pain group. In the unaffected side median nerve stimulation test, one patient showed activation of the ipsilateral SII, one patient of the contralateral primary somatosensory cortex SI, one patient of the bilateral SII, and one patient of the bilateral SII in the central neuropathic pain group.

In non-central neuropathic pain group, one patient showed activation of the contralateral SII, and one patient of the bilateral SII on the affected side, and two patients showed activation of the ipsilateral SI, one patient of the contralateral SI, three patients of the bilateral SII on the non-affected side.

\section{DISCUSSIONS}

The purposes of this study were to investigate the clinical aspects of central neuropathic pain, and to find an efficient screening tool for it. Four of seven thalamic stroke patients were assessed as having central neuropathic pain by the LANSS pain scale and the central neuropathic pain group and the non-central neuropathic pain group were not
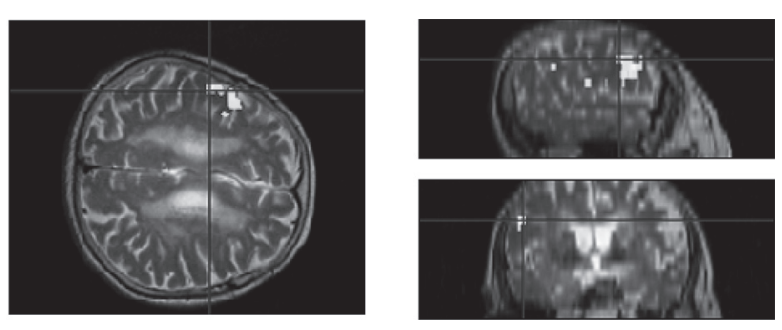

(a) The cortical activation map of a patient with central neuropathic pain
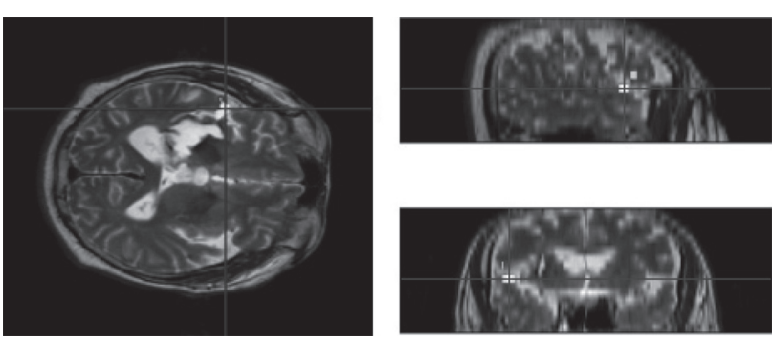

(b)

The cortical activation map of a patient with nonneuropathic pain

Fig. 1. The functional MRI cortical activation map induced by functional electrical stimulator

significantly different in quantitative sensory testing. Vestergaard et al. (1995) reported that the area of pain is within or less than that of the sensory deficit, which often shows allodynia or hyperalgesia ${ }^{8}$. The cardinal findings are that appreciation of pinprick and temperature is nearly always impaired $^{1,10)}$. and there is almost always a raised threshold to heat and cold detection and to a lesser extent also to painful heat and pain ${ }^{8,10)}$. There were differences 
between the result of this study and those of previous studies. In future studies, it will be necessary for generalization to use larger study groups to investigate the differences.

SEPs have been applied to assess the extent and location of acute damage and to predict the functional outcome of stroke patients ${ }^{1-13)}$. In this study, none of the enrolled patients showed SEPs in the median nerve stimulation on the affected side except one non-central neuropathic patient. However, all of the enrolled patients showed SEPs in median nerve stimulation on the unaffected side. The SEP results of this study did not discriminate well between the two groups because of the small sample size.

Previous studies employing anatomical and physiological methods demonstrate that the principal sensory nucleus (VP) and adjacent nuclei inferior and posterior to principal sensory nucleus in the thalamus are involved in nociceptive and thermal processing in monkeys and humans ${ }^{14-16)}$. In the present study, there were no differences in brain lesions in the MRI, and MRI as an independent measurement did not distinguish between neuropathic pain and non-neuropathic pain.

Although the relationship between stimulation frequency and volumes of cortical activation remains controversal, fMRI was used to determine patterns of cerebral blood flow changes in the somatosensory cortex induced by median nerve electrical stimulation ${ }^{17)}$. The role of SII has been proved in sensory discrimination demonstrating that SII is involved in and perceived pain in neuroimaging studies $^{18-20)}$. In the present study, SII cortical areas of the central neuropathic pain group were activated more sensitively than those of non-central neuropathic pain group in fMRI, indicating that fMRI is a meaningful tool for screening central neuropathic pain in thalamic stroke.

The limitations of this study were the small sample size, lack of an age-matched control group, and the performance of median nerve electrical stimulation without quantitative sensory stimulation or chemical stimulation in fMRI. We suggest that further research of central neuropathic pain in thalamic post stroke patients should consider the location and extent of lesion.

\section{ACKNOWLEDGEMENTS}

This research was supported by grant No. RTI04-01-01 from the Regional Technology Innovation Program of Ministry of Knowledge Economy (MKE).

\section{REFERENCES}

1) Leijon G, Boivie J, Johansson I: Central post-stroke pain-neurological symptoms and pain characteristics. Pain, 1989, 36: 13-25.

2) Bogousslavsky J, Regli F, Uske A: Thalamic infarcts: clinical syndromes, etiology and prognosis. Neurology, 1988, 38: 837-848.

3) Bowsher D: Central pain: clinical and physiological characteristics. J Neuril Neurosurg Psychiatry, 1996, 61: 62-69.

4) Walkins RH, Brody IA: The thalamic syndrome. Arch Neuril, 1969, 20: 559-562.

5) Hansson P: Post-stroke pain case study: Clinical characteristics, therapeutic options and long-term follow-up. Eur J Neurol, 2004, 11: 22-30.

6) Kim JS: Pure sensory stroke: clinical-radiological correlates of 21 cases. Stroke, 1992, 23: 983-987.

7) Kim JS: Lenticulocapsular hemorrhages presenting as pure sensory stroke. Eur Neurol, 1999, 42: 128-131.

8) Vestergaard K, Nielsen J, Andersen G, et al.: Sensory abnormalities in consecutive, unselected patients with central post-stroke pain. Pain, 1995, 61: 177-186.

9) Bennett M: The LANSS pain scale: The Leeds assessment of neuropathic symptoms and signs. Pain, 2001, 92: 147-157.

10) Boivie J, Leijon G, Johansson I: Central post-stroke pain-a study of the mechanisms through analyses of the sensory abnormalities. Pain, 1989, 37: 173-185.

11) La Joie WJ, Reddy NM, Melvin JL: Somatosensory evoked potentials: their predictive value in right hemiplegia. Arch Phys Med Rehabili, 1982, 63: 223-226.

12) Macdonell R, Donnan G, Bladin PF: Serial changes in somatosensory evoked potentials following cerebral infaction. Electroencephalogr Clin Neurophysiol ,1991, 80: 276-283.

13) Zeman BD, Yiannikas C: Functional prognosis in stroke: Use of somatosensory evoked potentials. J Neuro Neurosurg Psychiary, 1989, 52: 242-247.

14) Ohara S, Lenz F: Medial lateral extent of thermal and pain sensations evoked by microstimulation in somatic sensory nuclei of human thalamus. $\mathrm{J}$ Neurophysiol, 2003, 90: 2367-2377.

15) Apkarian AV, Shi T: Squirrel monkey lateral thalamus. Somatic nociresponsive neurons ans their relation to spinothalamic terminals. J Neurosci, 1994, 14: 6779-6795.

16) Lenz FA, Dougherty PM. Cells in human principal thalamic sensory nucleus (ventralos caudalis- $\mathrm{Vc}$ ) respond to innocuous mechanical and cool stimuli. J Neurophysiol, 1998, 79: 2227-2230.

17) Boakye M, Huckins SC, Szeverenyi NM, et al.: Functional magnetic resonance imaging of somatosensory cortex activity produced by electrical stimulation of the median nerve or tactile stimulation of the index finger. J Neurosug, 2000, 93: 774-783.

18) Bornhovd K, Quante M, Glauche V, et al.: Painful stimuli evoked different stimulus-responses functions in amygdale, prefrontal, insula and somatosensory cortex; a single-trial fMRI study. Brain, 2002, 125: 13261336 .

19) Coghill RC, Sang CN, Maisog JM, et al.: Pain intensity processing within the human brain: abilateral, distributed mechanism, J Neurophyiol, 1999, 82: 1934-1943.

20) Ploner M, Schmitz F, Freund HJ, et al.: Parallel activation of primary and secondary somatosensory cortices in human pain processing. J Neurophysiol, 1999, 81: 3100-3104. 